\title{
Electrophysiological Characterization of Spontaneously Contracting Cell Aggregates Obtained from Rainbow Trout Larvae with Multielectrode Arrays
}

\author{
Julia M. Mehnert ${ }^{a} \quad$ Matthias Brandenburger ${ }^{a, c} \quad$ Bianka Grunow ${ }^{a, b, c}$ \\ a Fraunhofer Research Institution for Marine Biotechnology EMB, Lübeck, Germany; \\ b Present address: University of Manchester; Faculty of Life Sciences, Core Technology Facility, \\ Manchester, UK; ${ }^{c}$ These authors contributed equally to this work
}

\section{Key Words}

Electrophysiology • Cardiac model system • Pharmacology • QT drugs

\begin{abstract}
Background/Aims: Safety pharmacology requires novel model systems for the detection of cardiac side effects. Ranging from cell-based systems to model organisms, no model available to date reflects the complexity of the human heart and evokes the great need for improved and more affordable systems. Many drugs interact with hERG potassium channels and consequently cause life threatening ventricular arrhythmias, further highlighting the importance of suitable model systems. Methods: Spontaneously Contracting Cell aggregates (SCC) as a 3D in vitro heart-syncytium obtained from rainbow trout larvae represent a novel model system for cardiac safety pharmacology. SCCs can be harvested cost-effectively and kept in culture for several weeks while retaining their functionality and displaying contraction rates similar to the human heart. Results: Extracellular field potential recordings with multielectrode arrays revealed significant prolongation of field potential duration upon administration of common hERG potassium channel blockers. Infusion of $1 \mu \mathrm{M}$ Dofetilide and $10 \mu \mathrm{M}$ Terfenadine prolonged field potentials 10 fold and 2 fold, respectively. In addition, SCCs enabled analysis of autonomous contraction frequencies. Conclusion: Thus, SCCs represent a novel and lowcost cardiac model system of the human heart for application in safety pharmacology.
\end{abstract}




\section{Introduction}

Pharmacological research and the development of new cardiac medicines is an important field of research and an increasing sector. However, the use of novel drugs and chemicals requires reliable data on the potential toxic side effects on the heart. Therefore, many research groups have recently tried to develop model systems for the use in human cardiac safety pharmacology. These model systems scale up from cell-based systems to model organisms. To date, there is no model available which is low-cost and accurately reflects the complex electrophysiology of the human heart.

\section{Development of drugs and the ERG (Either-a-gogo-Related-Gene) channel}

The average cost of developing a new medication is approximately 802 Million US\$ and takes 12 years [1] at which point the preclinical studies already cost 315 Million US\$ [2]. Despite very high acceptance standards and costs, many new drugs fail at the preclinical stage or are withdrawn from the market because targets validated using current in vitro models prove to be unreliable in humans. Therefore, more than 100 cardiac and noncardiac drugs are on the QT Drug List (e.g. Cisapride, Ephedrine, Cloroquine, Dofetilide or Terfenadine; QT Drug List, Arizona CERT [3]). These drugs may cause a specific type of ventricular arrhythmia, termed drug-induced torsades tachycardia. This arrhythmia is caused by inhibition of the rapid delayed rectifier current $\left(\mathrm{I}_{\mathrm{Kr}}\right)$ and is associated with a prolongation of the QT interval on the electrocardiogram [4].

In 1997 the CPMP (Committee for Proprietary Medicinal Products) released the guideline "Points to consider: The assessment of the potential for QT interval prolongation by non-cardiovascular medicinal products" for preclinical and clinical drug studies [5]. Since then, there is a consensus about the importance of drug interactions with $\mathrm{I}_{\mathrm{Kr}}$ ERG potassium channels [6]. In 2005, the ICH (International Conference on Harmonisation) published the guideline S7B for nonclinical evaluation of the potential for QT prolongation by human pharmaceuticals [7]. The S7B explicitly called for the use of in vitro $\mathrm{I}_{\mathrm{Kr}}$ assays.

An ERG channel block leads to a delayed repolarization current which promotes a prolonged field potential duration (FPD), which is reflected by the QT prolongation. For the human ERG channel, Mitcheson et al. identified specific amino acids by alanine-scanning mutagenesis, which represent binding sites for drugs comprising aromatic structures [8]. These amino acids are located in the S6 transmembrane segment (G648, Y652, and F656) and the pore helix (T623 and V625). The aromatic amino acids among them are unique to ERG potassium channels providing a structural explanation for the blocking of the ERG channel but no other voltage gated ion channel.

As a result, one of the aims of safety pharmacology studies is the detection of potentially undesirable effects of the tested drug in very early stages of preclinical development.

\section{Model systems}

For the examination of drugs, both in vitro and in vivo models are classically used. In vitro models are mostly used in phase I of preclinical development while in vivo models are used in later stages. Most of the common in vitro models in cardiotoxicology are primary isolated cells from human heart [9]. Further, popular cell based models are Human Embryonic Kidney (HEK) and Chinese Hamster Ovary (CHO). In these cell lines, the human ERG channel is transfected and the cells are used for patch clamp [10]. However, these models are limited by an absence of cell-cell-interactions and imperfect metabolism [11]. Other common in vitro models are established from rodents, such as mouse. A blocking of the ERG potassium channel cannot be examined in murine cell models due to a different expression of cardiac proteins and the absence of this channel in the mouse heart $[12,13]$. Consequently, there is a great need for replacement or supplementation of current in vitro models with new, improved and more affordable high-throughput models. 
Fish based test systems

Several working groups have recently promoted the use of fish as a cardiac model system in phase I of preclinical development (e.g. [14-16]. Fish action potentials, like humans, have a fast depolarization and a plateau phase followed by a repolarization. Grunow et al. developed a cost effective in vitro model based on fish myocardial cells. This in vitro model, called Spontaneously Contracting Cell aggregates (SCC), is a 3D in vitro "heart-syncytium" which beats and remains stable for more than 60 days $[16,17]$. The SCCs exhibit the typical cardiac markers, such as troponin I, connexin 43 , myosin heavy chains as well as compelling electrophysiological evidence of their suitable drug discovery [16]. Further, this inexpensive model could be generated very fast and in high numbers. Grunow et al. generated more than one SCC from one rainbow trout larva [18]. In the present study, the presence and function of ERG channels in rainbow trout SCCs are measured using extracellular recordings to establish the suitability of this model in preclinical drug development.

\section{Materials and Methods}

\section{Generation of SCCs}

SCCs were generated from rainbow trout larvae (Oncorhynchus mykiss, Walbaum) in the eye-point stage procured from fish farms in Born/Darß (Germany), and subsequently kept indoors at $6^{\circ} \mathrm{C}$. Cell isolation was performed from larvae shortly before hatching as published before by Grunow et al. [16] (Fig. 1a). Manual dissection using scissors was carried out in $0.1 \%$ trypsin/EDTA $(5.0 \mathrm{mg} / \mathrm{ml}$ Trypsin, $2.2 \mathrm{mg} / \mathrm{ml}$ EDTA, PAA Laboratories, Austria) for one minute. Digestion was stopped by adding the triple volume of cell culture medium (DMEM (Dulbeccos Modified Eagle Medium, Gibco, Gemany), 20 \% FCS (fetal calf serum, PAA Laboratories, Austria), $100 \mathrm{U} / \mathrm{ml}$ Penicillin and $0.1 \mathrm{mg} / \mathrm{ml}$ Streptomycin (PAA Laboratories, Germany)) and centrifugation for $5 \mathrm{~min}$ at $130 \mathrm{~g}$. Cell pellets were resuspended in $2 \mathrm{ml}$ cell culture medium and seeded into 6 well culture plates (Techno Plastic Products AG, Switzerland). In case of contaminations antibiotics (Gentamycin: $100 \mu \mathrm{g} / \mathrm{ml}$, Kanamycin: $100 \mu \mathrm{g} / \mathrm{ml}$ ) and antimycotics (Partricin: $0.5 \mu \mathrm{g} / \mathrm{ml}$ ) were added. Cells were cultivated at $20^{\circ} \mathrm{C}$ and $1.9 \% \mathrm{CO}_{2}$ while SCCs started to develop (Fig. 1b). The medium was changed two days after preparation and subsequently twice a week.

\section{Immunohistochemistry}

SCCs were embedded in Tissue-Tek ${ }^{\circledR}$ (Sakura Finetek), frozen at $-80^{\circ} \mathrm{C}$ and cryo-sliced into $5 \mu \mathrm{m}$ tissue slices. The tissue was fixed with methanol:acetone $\left(7: 3\right.$, kept at $-20^{\circ} \mathrm{C}$ ) containing $1 \mathrm{mg} / \mathrm{ml}$ DAPI (Roche, Switzerland) for $5 \mathrm{~min}$ at $20^{\circ} \mathrm{C}$. After $20 \mathrm{~min}$ incubation with $1.7 \%$ normal goat serum (Vector Laboratories, USA) at $20^{\circ} \mathrm{C}$, primary antibody (polyclonal rabbit anti hERG, 1:40, Sigma-Aldrich, Germany) or IgG control (rabbit IgG, 1:16, Santa Cruz Biotechnology, USA) was incubated for $2 \mathrm{~h}$ in a humid chamber at $37^{\circ} \mathrm{C}$. The antibody was targeted against the C-terminal end of ERG with reactivity proven against human, rat and mouse. Secondary antibody (FITC conjugated goat anti rabbit, 1:200, Dianova, Germany) was incubated in a humid chamber for $1 \mathrm{~h}$ at $37^{\circ} \mathrm{C}$. Washing steps were carried out with DPBS (Dulbecco's Phosphate buffered saline, Gibco Invitrogen, Germany). The slides were covered in Vectashield ${ }^{\circledR}$ (Vector Laboratories, USA) and analyzed with a laser scanning microscope (LSM T-PMT, Zeiss, Germany).

\section{Sequence alignments}

Sequence entries obtained from the protein database UniProt were used for sequence alignments [19]. Accession number hERG: UniProt: Q12809, accession number ERG O. mykiss: UniProt: A7LA95. Alignments were carried out using the UniProt service program.

Detection of electrophysiological parameters

A multielectrode array (MEA, System and equipment obtained from Multi Channel Systems, Germany) was used to obtain electrophysiological recordings. Glass MEA chips had 60 integrated titanium nitride electrodes with a diameter of $30 \mu \mathrm{m}$ and an electrode-distance of $200 \mu \mathrm{m}$. An attached plastic ring created a chamber and enabled cultivation in 1-2 ml medium. This defined area of MEA chips was coated with pork gelatine $\left(0.1 \%(\mathrm{w} / \mathrm{v})\right.$, Fluka, Switzerland) for $30 \mathrm{~min}$ at $37^{\circ} \mathrm{C}$ to improve cell attachment. 


\section{Cellular Physiology $\quad$ Cell Physiol Biochem 2013;32:1374-1385 and BiOchemistry \begin{tabular}{l|l} 
DOI: 10.1159/000356576 & Published onlıne: November 22, 2013 \\
\hline
\end{tabular} \\ Mehnert/Brandenburger/Grunow: Electrophysiology of Spontaneously Contracting Cell Aggregates}

Fig. 1. SCCs obtained from rainbow trout larvae as a novel cardiac model system a) Rainbow trout larvae (eye-point stage) before preparation b) Developed SCC in a cell culture plate c) SCC adhered to a MEA-chip covering 11 electrodes d) MEA-chip during pharmacological measurement, inflowand outflow tubes allow continuous perfusion with pharmacological substances.

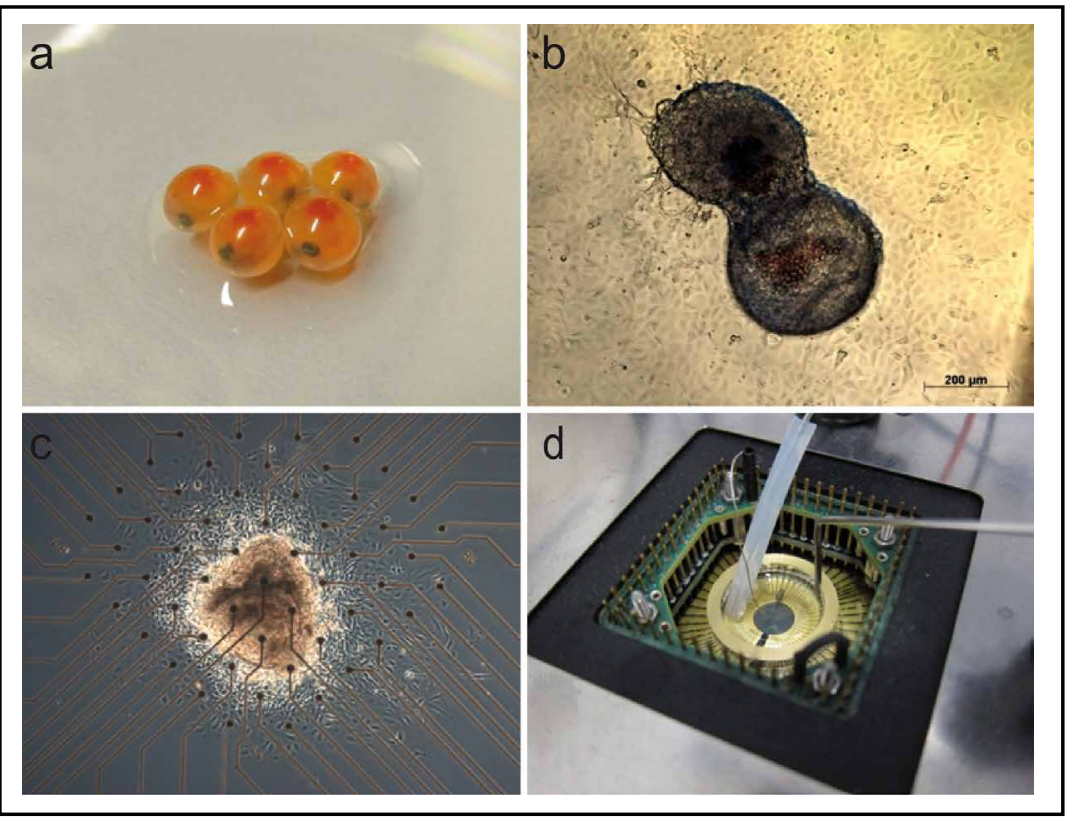

Table 1. Dofetilide and Terfenadine: Times of addition and corresponding concentrations (L-15: Leibovitz's L-15 medium)

\begin{tabular}{|c|c|c|c|c|c|c|c|c|c|}
\hline Time (min) & 0 & 20 & 30 & 40 & 50 & 60 & 70 & 80 & 90 \\
\hline \multicolumn{10}{|l|}{ Dofetilide } \\
\hline Concentration (nM) & L-15 & 10 & 100 & 200 & 400 & 600 & 800 & 1000 & L-15 \\
\hline \multicolumn{10}{|l|}{ Terfenadine } \\
\hline Concentration (nM) & L-15 & 100 & 1000 & 2000 & 4000 & 6000 & 8000 & 10000 & $\mathrm{~L}-15$ \\
\hline
\end{tabular}

Isolation of SCCs for electrophysiological measurements was performed using a scalpel and pipette. SCCs were used in the course of two months after preparation. After transfer to MEA chips SCCs were placed upon electrode endings and cultured in $1 \mathrm{ml}$ cell culture medium at $20^{\circ} \mathrm{C}$ and $1.9 \% \mathrm{CO}_{2}$. After $\sim 2-3$ days SCCs attached (Fig. 1c) and could be used for electrophysiological recordings and pharmacological measurements. SCCs were attached for at least one day and usually covered about 2-6 electrodes on the MEA chip. Measurements were carried out at $20^{\circ} \mathrm{C}$ without $\mathrm{CO}_{2}$-gassing and under perfusion with L-15 medium (Leibovitz's L-15 medium, Gibco, Germany).

Field potentials (FPs) detected by the integrated electrodes were transmitted to an amplifier and then visualized by the associated software MC_Rack. Recordings ran for 10 min per measurement before data for electrophysiological parameters (contraction frequency and FPD) were collected.

\section{Pharmacological measurements}

For pharmacological measurements, inflow and outflow tubes were used to allow continuous perfusion with pharmacological substances (Fig. 1d). Substances were dissolved in DMSO (0.1\% DMSO for Dofetilide, $0.01 \%$ DMSO for Terfenadine) and diluted in L-15 medium to set the particular concentrations. Before adding substances, the system with the attached SCCs was perfused with pure L-15 medium for 20 min for adaptation. Following the last concentration of the tested substance, perfusion with L-15 medium was carried out for 20 min to survey any effect accompanying the substance wash-out.

The appropriate range of concentrations to establish dose response curves was determined for each applied substance (Table 1).

\section{Data analysis}

During pharmacological measurements with the MEA, the software MC_Rack saves and visualizes received data in real-time (Fig. 2a). Data used for dose response curves and further analyses were obtained 


\section{Cellular Physiology $\quad$ Cell Physiol Biochem 2013;32:1374-1385 and Biochemistry

Fig. 2. Visualization of field potentials by the software MC_Rack a) Since the SCC covers 3 electrodes, only these and surrounding electrodes are selected. b) The electrode providing the most distinct signal is displayed.

data analysis, peaks were detected and plotted as a function of time.
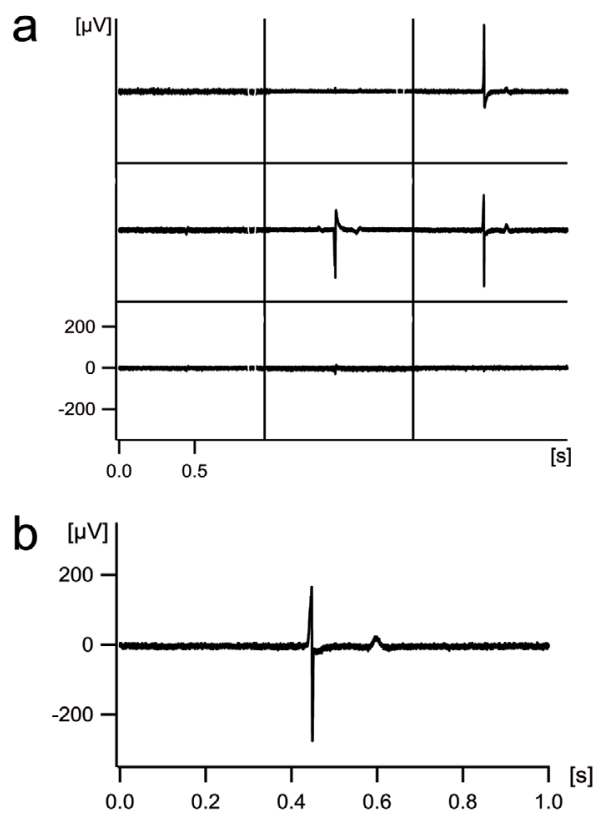

from one electrode on the MEA chip providing the most distinct signal (exemplary shown in Fig. 2b). For

FPD was analyzed by detecting the distance from the first prominent peak in the FP to the following maximum or minimum of the repolarization wave.

Contraction frequency was detected by measuring the temporal occurrence of prominent peaks initiating each FP.

For quantified comparisons between FPD and contraction frequency, two points of time were selected. The first was chosen just before addition of the lowest drug concentration and the second point was chosen when the maximum contraction rate was reached.

\section{Statistical analysis}

Data are presented as mean \pm SEM. From all recorded data points of one experiment, the last five data points before adding the next concentration were selected for analyses using Microsoft Excel 2010. Differences between untreated and treated samples were evaluated using a Student's t-test (paired, doublesided) with Prism 5.04 (Prism ${ }^{\circledR}$ GraphPad Software). The confidence interval was set $95 \%$. Prism was applied for calculating SEM, $\mathrm{EC}_{50}$-values and for curve fitting (nonlinear fit, variable slope; Fitting method: Least squares fit).

\section{Results}

\section{General SCC characteristics}

After their isolation, cells attached within 1-2 days. Cell monolayers as well as threedimensional aggregates were visible. SCCs were detectable after about two days. SCCs, which appeared in suspension, partially attached after transfer to another culture plate. Preparations of larvae provided yields between $65 \%$ and $88 \%$. Characteristics of SCCs match the published data by Grunow et al. [18]. Round SCCs with one contraction center were detected as well as SCCs with two contraction centers. Contractions appeared less frequently than once per second and often the direction of contraction was visible (exemplary video: $\mathrm{ftp}: / /$ cetres.emb.fraunhofer.de/cetres/scc/Supplements_SCC_Video.mp4). The diameter of recently developed SCCs was 200-600 $\mu \mathrm{m}$ and growth was observed during cultivation up to a doubling in size. 
Mehnert/Brandenburger/Grunow: Electrophysiology of Spontaneously Contracting Cell Aggregates

Fig. 3. The ERG potassium channel in SCCs a) The hERG potassium channel with six transmembrane segments and the pore opening between segment five and six. Alignment was carried out with amino acid sequences of the pore opening and transmembrane segment six between hERG and ERG of Oncorhynchus mykiss. Amino acids which participate in drug-channel-interactions (G648, Y652, and F656 in the S6 transmembrane domain; T623 and V625 in the pore helix) are marked with green boxes [1]. b) Immunohistochemical staining on SCC cryo-slices with an antibody against hERG reveals distinctive fluorescence at the cell membrane (green). Nuclei are detected with DAPI (blue) c) Zoomed detail.

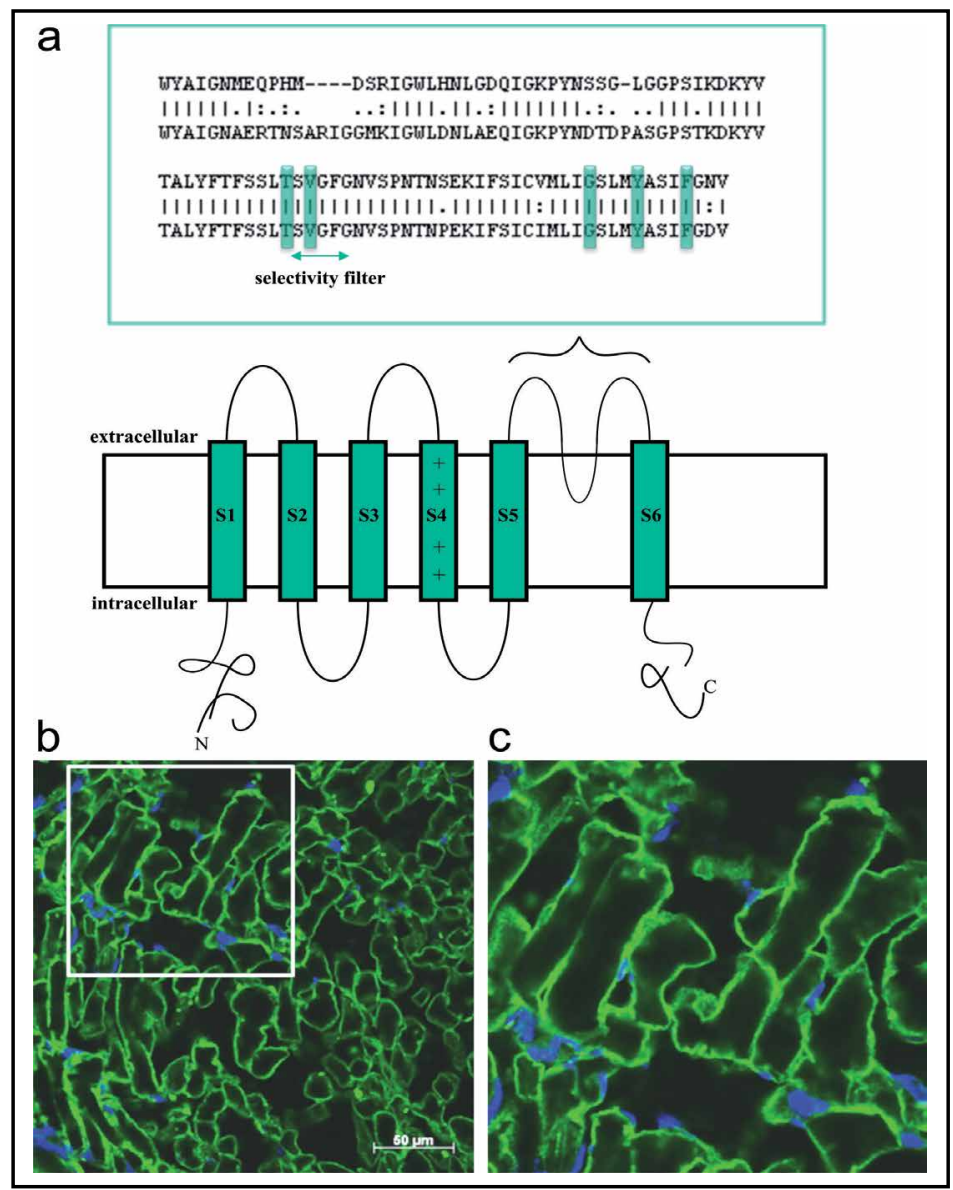

Following the transfer to MEA chips for pharmacological measurements, a temporary absence of contractions was observed in some cases. After at least one day contractions restarted and remained constant.

Growth of SCCs attached to MEA chips was homologous to growth in standard cell culture plates.

An accurate determination of contraction frequency and FPD $(n=12)$ was carried out. The recorded FPs revealed depolarization and repolarization currents and thus the FPD. The average contraction rate was $0.6 \pm 0.05 \mathrm{~Hz}$ and the average FPD was $170.2 \pm 13.01 \mathrm{~ms}$. Amplitude of peaks in recorded FPs (depolarization peak) ranged from about 100 to $400 \mu \mathrm{V}$.

\section{ERG potassium channel in SCCs}

An alignment between human ERG and ERG of rainbow trout (O. mykiss) was carried out to identify possible sites of drug interaction in the new model system. The alignment, performed with complete ERG protein sequences of both species, revealed identity of $54.3 \%$. In the sequence of 92 amino acids representing the pore opening and the S6 transmembrane segment, the identity was even $74.2 \%$. The critical amino acids mentioned in the introduction could be identified in this sequence in ERG of both species (Fig. 3a).

Results of the alignment are based on the genome of fully developed rainbow trout. Further experimental verification to adopt these results on SCCs, which are of larval origin, was carried out.

Immunohistochemical stainings gave further evidence of an ERG homologue in SCCs. The membrane-located fluorescence (Fig. $3 b$ and 3c) indicates the presence of an ERG homologue in addition to alignments. A control staining only with secondary antibody was negative and also an IgG control, used for standardization of fluorescence intensity, showed no signal (unpublished data). 


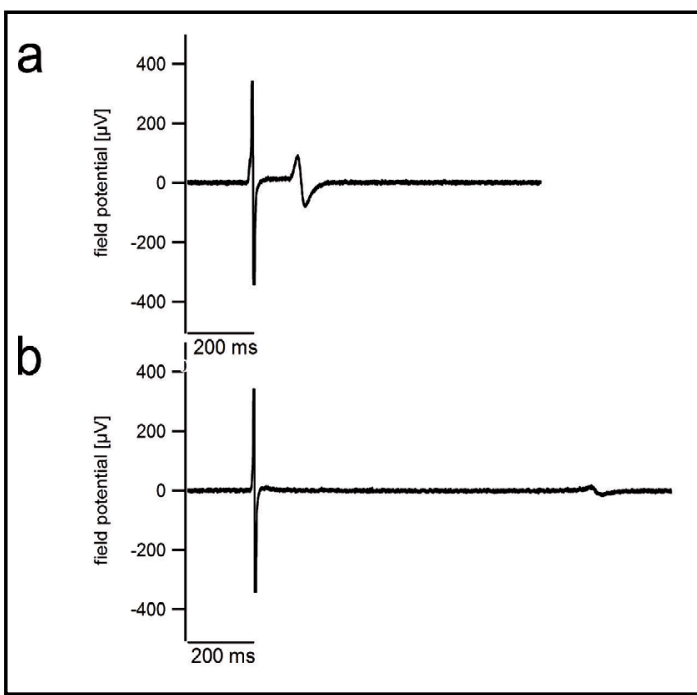

Fig. 4. Field potential prolongation a) A normal FP of one SCC under perfusion with L-15 medium is recorded with MEA b) addition of $1000 \mathrm{nM}$ Dofetilide prolongs the FP by shifting the repolarization wave to the right.

Fig. 5. Effects of Dofetilide and Terfenadine on FPD a) in comparison with untreated control and solvent ( $\mathrm{n}=3-10)$. b) Dose response curve of Dofetilide $(\mathrm{n}=3) \mathrm{c}$ ) Dose response curve of Terfenadine $(n=3)$. Student's t-test: $n . s .:$ not significant, $p>0.05$, ${ }^{*}: \mathrm{p}<0.05,{ }^{* *}: \mathrm{p}<0.01$, data \pm SEM.

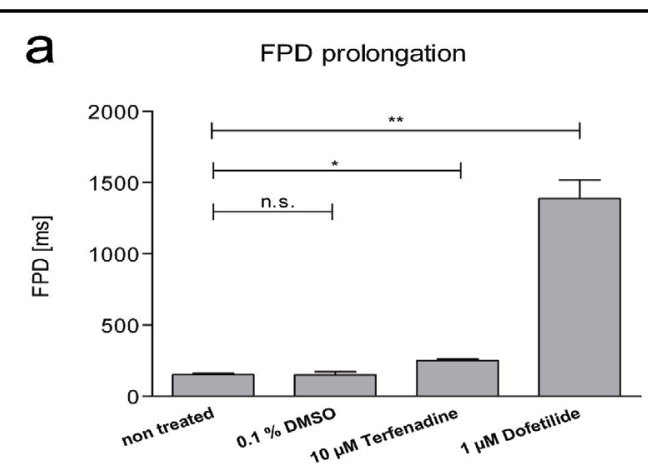

b

Dofetilide

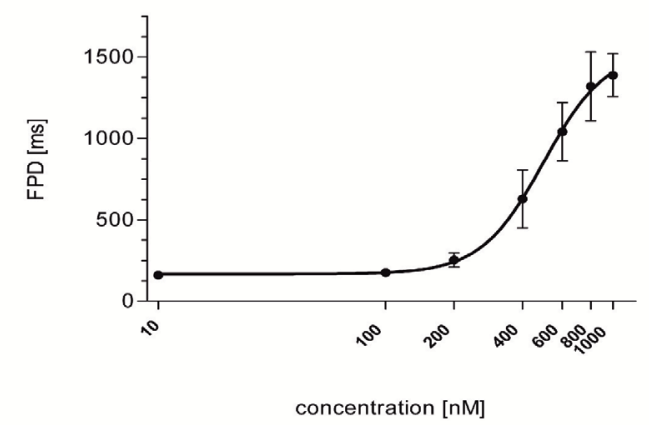

C

Terfenadine

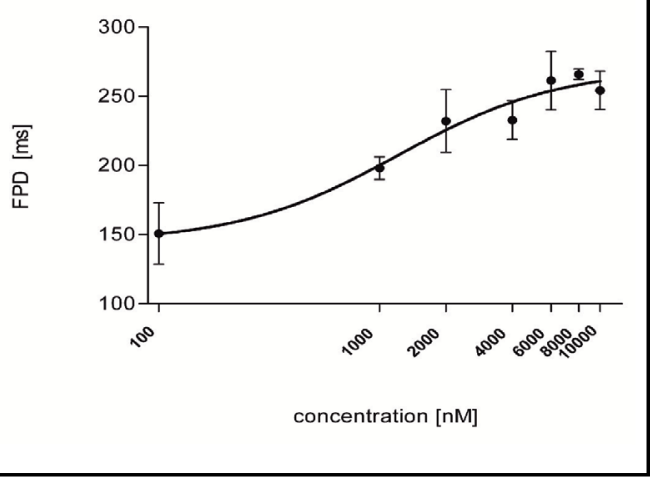

Effects of $h E R G$ blockers Dofetilide and Terfenadine on SCCS

Effects on FPD. After 20 minutes of L-15 perfusion, SCCs adapted to the new environment and had stable FPs. The FP prolonging effect of drugs was measured during the whole measurement. The exemplary FPD is displayed for Dofetilide in Fig. 4.

Dose response analyzes for Dofetilide and Terfenadine reveal a 9 fold and 2 fold maximum FPD, respectively (Fig. 5). The increase occurs with dose dependency. For Dofetilide, the prolongation from $160 \mathrm{~ms}$ to $1400 \mathrm{~ms}$ was significant $(\mathrm{p}<0.01)$ and the prolongation from $140 \mathrm{~ms}$ to $250 \mathrm{~ms}$ was significant for Terfenadine ( $\mathrm{p}<0.05$ ), compared to untreated SCCs (each substance: $\mathrm{n}=3$ ). When measurements were completed, it was not possible to see any effect of washing out the substances.

DMSO as a solvent for Dofetilide and Terfenadine was tested for possible FP prolongation. The higher applied concentration of $0.1 \%$ DMSO in Dofetilide measurements did not show any effect on FPD $(p>0.05)$.

The dose response curve for Dofetilide had an $\mathrm{EC}_{50}$-value of $469.4 \pm 74.8 \mathrm{nM}$.

Effects on contraction rate. The contraction rate was recorded simultaneously with FPD in the same measurement. This analysis enabled discovery of potential correlations between the two parameters. 


\section{Cellular Physiology $\quad$ Cell Physiol Biochem 2013;32:1374-1385 and Biochemistry

Fig. 6. Data of one measurement with Dofetilide perfusion a) Field potential duration (left axis) and contraction rate (right axis) are plotted against time, in which increasing concentrations of Dofetilide are added. Simultaneously to the first prolongation of field potential duration, contraction rate increased suddenly and decreases afterwards. Two points of time are tagged: Point before addition of Dofetilide ( $\mathrm{t}-(\mathrm{L}-15))$ and point of maximum rate $\mathrm{t}(\mathrm{Max}$ rate)) b) Field potential duration and contraction rate are analyzed at $\mathrm{t}$-(L15) and at $t$ (Max rate). $\mathrm{n}=3$, Student's t-test: *: $\mathrm{p}<0.05, * *: \mathrm{p}<0.01$, data \pm SEM.

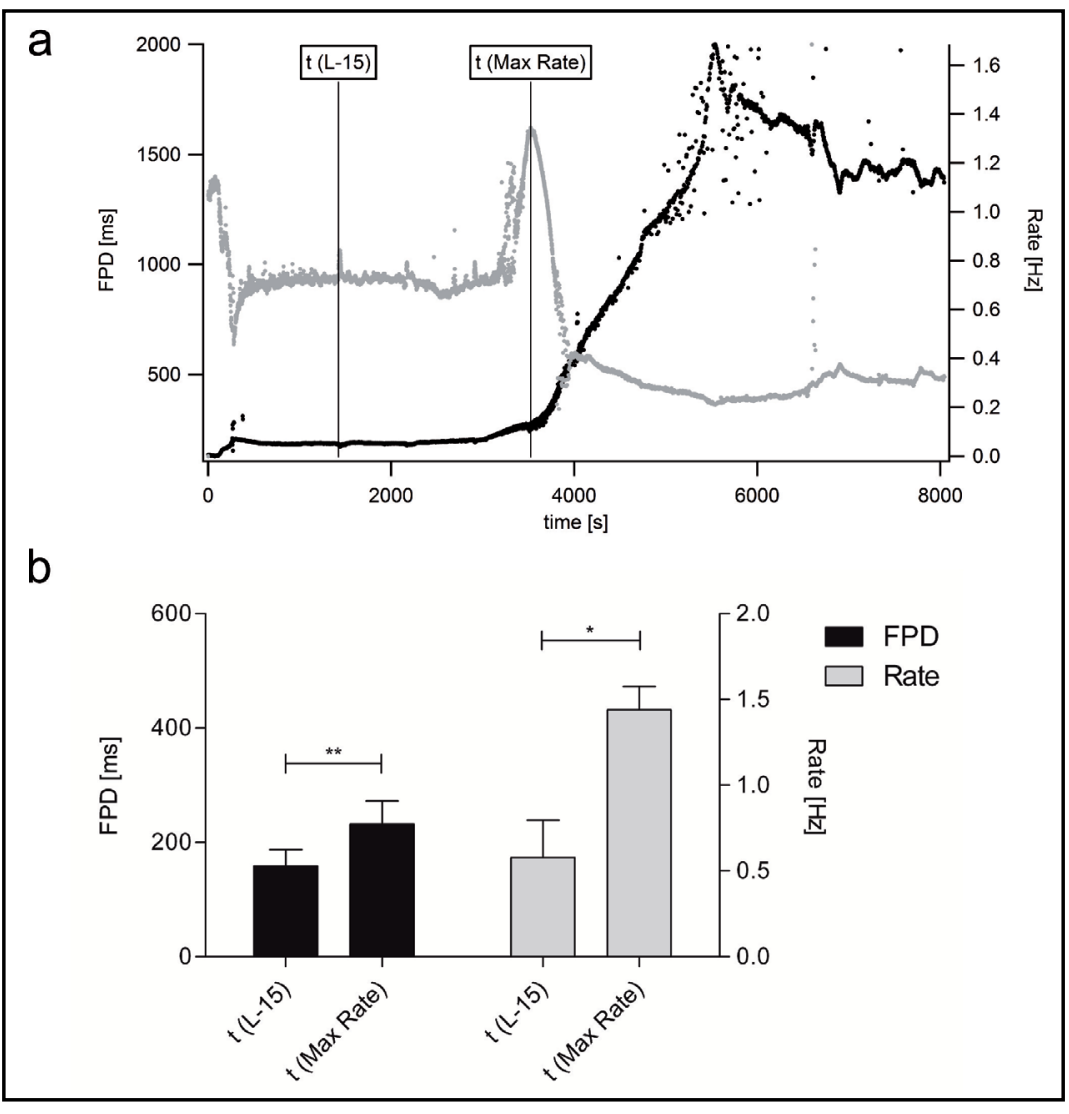

In general, due to prolonged refractory periods, contraction rate of the SCCs decreased with higher concentrations of either drug which led to FP prolongation.

Extended time periods under drug perfusion and high drug concentrations influenced the contraction rate in later stages of the experiment and even led to the absence of contraction.

A striking observation was made during pharmacological measurements with Dofetilide; with the onset of the first detectable prolongation of FP at a concentration of approximately $200 \mathrm{nM}$, the contraction rate rose rapidly and its values more than doubled (Fig. 6a, n=3). During addition of the next higher concentration, contraction rate decreased again, even reaching values lower than before the rapid increase.

To quantify this observation, contraction rate and FPD were compared to each other at two points in time (see Methods). FPD and contraction rate increased significantly between the compared times (Fig. 6b).

For Terfenadine, simultaneously recorded contraction rates did not show any increase in contraction rate $(n=3)$.

\section{Discussion}

Combining high data quality, high throughput and low costs in one cardiac model system is the optimal scenario for preclinical safety pharmacological studies, but this remains a great challenge.

The pharmacological characterization of a novel model system requires stringent tests which confirm its applicability. For cardiac safety pharmacology, these tests include the detection of drug-induced ERG block and the potential drug influence on contraction rate. As many drugs cause undesirable QT prolongation and are subsequently withdrawn from the market, it is crucial to detect adverse effects as early as possible in a model system. This 
torsade tachycardia, often linked to QT prolongation, must be identified early in preclinical studies.

\section{Contraction rate and FPD in SCCs}

Two characteristic parameters, contraction rate and FPD (reflecting QT interval), were statistically analyzed in rainbow trout SCCs. The comparison of the data to mouse and human enabled the classification of our novel model system. Contraction frequency and QT interval of SCCs are more similar to human than mouse to human parameters. The contraction frequency of rainbow trout SCCs differs from human by a factor of 2 , whereas mouse differs from human by a factor of 8.3. With respect to QT interval, SCCs differ from human by a factor of 2.7 while mouse differs from human by a factor of 5.1 [20-22]. In addition to previously collected data on typical cardiac proteins [16], long-term and stable contractions [18] and molecular analyses, our present data confirms the suitability of SCCs as a model system for the human heart.

\section{Evidence of ERG in SCCS}

A sequence alignment was carried out to identify similarities between the human ERG and ERG of 0 . mykiss. The entire sequence identity of $54.3 \%$ is comparatively low; mouse and rat ERG sequences show $>97 \%$ identity to human ERG [23]. Although there is a lower sequence identity, there must be conserved regions, such as selectivity filter or structureproviding sequences, enabling potassium channel functionality.

It was possible to select specific regions in which interaction with substances could be observed. These are regions in the pore opening and S6 transmembrane segment, comprising some conserved amino acids mentioned in 1.1 (T623, V625, G648, Y652 and F656) [8, 24]. In this area, an identity of $74.2 \%$, and identification of the amino acids mentioned above, indicates a high probability for an identical reaction towards ERG blockers.

Assumptions of a similar reaction towards ERG blockers are based on fully developed organisms so far. The immunohistochemical stainings should show the presence of ERG in SCCs, which are not obtained from fully developed but from larval origin. Signals (Fig. 3b) revealed existence of an ERG related channel in SCCs. There must be high structural identity in the $\mathrm{C}$-terminal region, the binding epitope of the antibody.

\section{Detection of FPD prolongation}

SCCs clearly showed a drug-induced FP prolongation, which represents a QT prolongation due to linear dependency [25]. Application of Dofetilide and Terfenadine resulted in a 9 fold and 2 fold prolongation, respectively. These data show the potential of SCCs to be used for identification of QT prolonging drugs. However, concentrations used for therapy in patients as well as human-specific $\mathrm{EC}_{50}$ values cannot be directly compared to concentrations applied in the model system. The differences in size and species-characteristic temperatures naturally give rise to distinctions based on the different species.

For example, a temperature increase from room temperature $\left(20-24^{\circ} \mathrm{C}\right)$ to body temperature $\left(36-37^{\circ} \mathrm{C}\right)$ has an impact on gating characteristics and ERG channel kinetics [26] as well as drug-receptor binding [27].

Because of highly different dimensions between the human organism and SCCs as model system, the high reacitivity of SCCs towards hERG blockers is a notable feature. The ability of a model in vitro heart-syncytium to react like the human heart in this issue is particularly convenient for safety pharmacological studies.

\section{Detection of contraction frequency}

In our model system we were able to detect a sudden increase of contraction frequency during Dofetilide measurements with onset of the first detectable FPD prolongation. This observation gives an indication of the drug's side effect in patients.

Dofetilide, a class III antiarrhythmic, aims to convert atrial fibrillation into a stable sinus rhythm by prolonging the QT interval [28]. Evoking the opposite, in the DIAMOND study [29] 
$3.3 \%$ of patients suffered from life threatening torsade tachycardia. Torp-Pedersen et al. reported sudden onset of torsade tachycardia in parallel to the therapeutic QT prolongation, in which normal doses of Dofetilide were applied [30]. These clinical studies confirm that SCCs are a model system for estimating the hazardous risks of tachycardia.

In Terfenadine measurements, no sudden increase in contraction rate was observed in rainbow trout SCCs. This is in correlation with clinical studies in which the onset of torsade tachycardia only occurred when overdosed or in combination with other drugs [31]. Case studies explain the risk of overdosing and combining Terfenadine with other drugs, like Ketoconazole [32, 33].

To conclude, in our model system Dofetilide and Terfenadine evoked effects that were similar to those observed in clinical studies with administration of therapeutic doses.

\section{Competitiveness of SCCS}

For a novel cardiac model system to be competitive, it must reduce costs with consistent data quality or improve data quality with consistent costs and a good throughput.

The individual pharmacological measurement of each SCC decelerates the procedure of multiplex drug measurements. Because of fast and easy production of SCCs, the throughput is much higher than whole hearts or heart slices, but is not to be ranked as high-throughput yet. We place the throughput rate of SCCs on 48 data points per day, which is a comparable value to other systems such as patch clamp on transfected HEK or CHO cells or repolarization assays on papillary muscles or purkinje fibers [1]. In comparison to heart slices, whole hearts or organ preparations like purkinje fibers, SCCs don't depend on clinical sample material but enable effectively timed coordination of measurements.

Human heart slices provide higher data quality, but cannot be used in medium - or high - throughput [34].

One major benefit of our system is the many similar features compared to the human heart. Besides the similar contraction frequency, there is the existence of specific cardiac proteins as well as gap junctions resulting in a functional cardiomyocyte-syncytium [16]. Cell-cell interactions, compared to single cell systems requiring external stimulation, offer autonomous contractions and are therefore an attractive system for long-term investigations.

The data quality provided by SCC measurements can be classified as high compared to other systems. The exact detection of FPD, the potential of generating dose response curves and numerous quantifiable tools of data evaluation enable the detection of even slight shifts in FP form or length. Detection of conduction velocity is another additional feature which can be implemented.

Costs as an important economical factor are low compared to other established systems. After purchasing the multielectrode array, costs incurred are merely material costs plus personal expenditure. Currently, preparation of one larva and generation of SCCs costs less than $1 €[18]$.

Since the application of rainbow trout eggs doesn't concern animal experiments, ethical conflicts and any costs connected to permission of Protection of Animals Act do not apply.

\section{Perspectives}

SCCs are a promising model for industrial application in safety pharmacological studies. To provide even more data points per day at lower costs, it is possible to optimize the system. Similar to the test system developed by Meyer et al. [1], who use chicken embryos for cell isolation, our model can be scaled up to high-throughput. Measurements in multiwell dimensions are technically achievable and additional equipment is available by Multi Channel Systems (Reutlingen, Germany). With this improvement, costs will be lowered while still achieving higher throughput.

Additionally, an automatic protocol for disintegration of larvae will save time, increase the SCC generation rate and lower personal expenditure considerably.

Collectively, the improvements will accelerate the production process enormously and lower material and personnel costs. 
In conclusion, we classify rainbow trout SCCs as a high quality cardiac model system, which is low-cost and currently provides a good throughput rate. The system is suitable for identification of QT prolonging drugs and offers simultaneous recording of contraction frequency. The system has great potential for further improvements as costs can be reduced easily and an increase of throughput, even up to high-throughput, is also feasible.

\section{Abbreviations}

(h)ERG ((human) Either-a-gogo-Related-Gene); MEA (Multielectrode Array); SCC (Spontaneously Contracting Cell aggregate); FP(D) (Field Potential (Duration)).

\section{Conflict of Interest}

None declared

\section{Acknowledgements}

We are grateful to the Research Institute for Agriculture and Fishery in Born/Darß (Germany) providing the larvae for this study.

We also thank Carolin Wienke from the Fraunhofer Research Institution for Marine Biotechnology EMB for her technical support and Dr. Gina Galli from the University of Manchester for assistance in proof reading this manuscript.

This work was supported by the Ministry of science, economy and transport of Schleswig-Holstein and the European Community within the framework of the European Regional Development Fund (RDF).

\section{References}

1 Meyer T, Leisgen C, Gonser B, Günther E: QT-screen: high-throughput cardiac safety pharmacology by extracellular electrophysiology on primary cardiac myocytes. Assay Drug Dev Techn 2004;2:507-514.

2 Adams CP, Brantner VV: Estimating the cost of new drug development: Is it really 802 million? Health Affair 2006;25:420-428.

3 Azert: QT Drug List [cited November 2012]. Available from: http://www.azcert.org/medical-pros/druglists/drug-lists.cfm

-4 Yap YG, Camm AJ: Drug induced QT prolongation and torsades de pointes. Heart 2003;89:1363-1372.

5 The European Agency for the Evalution of Medical Products, editors. Guideline "Points to consider: The assessment of the potential for QT interval prolongation by non-cardiovascular medicinal products" for preclinical and clinical drug studies. Proceeding in Human Medicines Evaluation Unit [cited November 2012]. Available from: http://www.fda.gov/ohrms/dockets/ac/03/briefing/pubs/cpmp.pdf.

6 Redfern WS, Carlsson L, Davis AS, Lynch WG, MacKenzie I, Palethorpe S, Siegl PK, Strang I, Sullivan AT, Wallis R, Camm AJ, Hammond TG: Relationships between preclinical cardiac electrophysiology, clinical QT interval prolongation and torsade de pointes for a broad range of drugs: evidence for a provisional safety margin in drug development. Cardiovasc Res 2003; 8:32-45.

7 European Medicines Agency, editors. Guideline S7B for nonclinical evaluation of the potential for QT prolongation by human pharmaceuticals. Proceedings of the International Conference on Harmonisation [cited November 2012]. Available from: http://www.ema.europa.eu/docs/en_GB/document_library/ Scientific_guideline/2009/09/WC500002841.pdf.

8 Mitcheson JS, Chen J, Lin M, Culberson C, Sanguinetti MC: A structural basis for drug-induced long QT syndrome. Proc Natl Acad Sci USA 2000;97:12329-12333.

-9 Davidson MM, Nesti C, Palenzuela L, Walker WF, Hernandez E, Protas L, Hirano M, Isaac ND: Novel cell lines derived from adult human ventricular cardiomyocytes. J Mol Cell Cardiol 2005;39:133-147.

10 Thomas D, Wendt-Nordahl G, Röckl K, Ficker E, Brown AM, Kiehn J: High-affinity blockade of human ethera-go-go-related gene human cardiac potassium channels by the novel antiarrhythmic drug BRL-32872. J Pharmacol Exp Ther 2001;297:753-761. 
Mehnert/Brandenburger/Grunow: Electrophysiology of Spontaneously Contracting Cell Aggregates

11 Ausma J, Borgers M: Dedifferentiation of atrial cardiomyocytes: from in vivo to in vitro. Cardiovasc Res 2002;55:9-12.

12 Wang L, Feng ZP, Kondo CS, Sheldon RS, Duff HJ: Developmental changes in the delayed rectifier $\mathrm{K}^{+}$ channels in mouse heart. Circ Res 1996;79:9-85.

13 Pieperhoff S, Franke WW: The area composita of adhering junctions connecting heart muscle cells of vertebrates. VI. Different precursor structures in non-mammalian species. Eur J Cell Biol 2008; 87:413-30.

14 Brette F, Luxan G, Cros C, Dixey H, Wilson C, Shiels HA: Characterization of isolated ventricular myocytes from adult zebrafish (Daniorerio). Biochem Biophys Res Commun 2008;374:143-146.

15 Nemtsas P, Wettwer E, Christ T, Weidinger G, Ravens U: Adult zebrafish heart as a model for human heart? An electrophysiological study. J Mol Cell Cardiol 2010;48:61-171.

16 Grunow B, Wenzel J, Terlau H, Langner S, Gebert M, Kruse C: In vitro developed spontaneously contracting cardiomyocytes from rainbow trout as a model system for human heart research. Cell Physiol Biochem 2011;27:1-12.

17 Grunow B, Ciba P, Rakers S, Klinger M, Anders E, Kruse C: In vitro expansion of autonomously contracting, cardiomyogenic structures from rainbow trout Oncorhynchus mykiss. J Fish Biol 2010;76:427-434.

18 Grunow B, Schmidt M, Klinger M, Kruse C: Development of an in vitro cultivated, spontaneously and longterm contracting 3D heart model as a robust test system. J Cell Sci Ther 2012;3:116.

19 UniProt Browser [cited November 2012]. Available from: http://www.uniprot.org/

20 Dobson GP: On being the right size: heart design, mitochondrial efficiency and lifespan potential. Clin Exp Pharmacol P 2003;30:590-597.

21 Wehrens XH, Kirchhoff S, Doevendans PA: Mouse electrocardiography: an interval of thirty years. Cardiovasc Res 2000;45:231-237.

-22 Rautaharju PM, Surawicz B, Gettes LS, Bailey JJ, Childers R, Deal BJ, Gorgels A, Hancock EW, Josephson M, Kligfield P, Kors JA, Macfarlane P, Mason JW, Mirvis DM, Okin P, Pahlm O, van Herpen G, Wagner GS, Wellens: HAHA/ACCF/HRS Recommendations for the Standardization and Interpretation of the Electrocardiogram: Part IV: The ST Segment, T and U Waves, and the QT Interval A Scientific Statement From the American Heart Association Electrocardiography and Arrhythmias Committee, Council on Clinical Cardiology; the American College of Cardiology Foundation; and the Heart Rhythm Society Endorsed by the International Society for Computerized Electrocardiology. J Am Coll Cardiol 2009;53:982-991.

-23 Pond AL, Scheve BK, Benedict AT, Petrecca K, Van Wagoner DR, Shrier A, Nerbonne JM: Expression of distinct ERG proteins in rat, mouse, and human heart. Relation to functional I(Kr) channels. J Biol Chem 2000;275:5997-6006.

-24 Kamiya K, Niwa R, Morishima M, Honjo H, Sanguinetti M C: Molecular determinants of hERG channel block by terfenadine and cisapride. J Pharmacol Sci 2008;108:301-307.

-25 Halbach M, Egert U, Hescheler J, Banach K: Estimation of action potential changes from field potential recordings in multicellular mouse cardiac myocyte cultures. Cell Physiol Biochem 2003;13:271-284.

26 Wang J, Della Penna K, Wang H, Karczewski J, Connolly TM, Koblan KS, Bennett PB, Salata JJ: Functional and pharmacological properties of canine ERG potassium channels. Am J Physiol-Heart C 2003;284:H256-267.

27 Kirsch GE, Trepakova ES, Brimecombe JC, Sidach SS, Erickson HD, Kochan MC, Shyjka LM, Lacerda AE, Brown AM: Variability in the measurement of hERG potassium channel inhibition: effects of temperature and stimulus pattern. J Pharmacol Toxicol 2004;50:93-101.

-28 McClellan KJ, Markham A: Dofetilide: a review of its use in atrial fibrillation and atrial flutter. Drugs 1999;58:1043-1059.

29 The DIAMOND Study Group. Dofetilide in patients with congestive heart failure and left ventricular dysfunction. Danish Investigations of Arrhythmia and Mortality on Dofetilide Study Group. New Engl J Med 1999;341:857-865.

30 Torp-Pedersen C, Moller M, Kober L, Camm AJ: Dofetilide for the treatment of atrial fibrillation in patients with congestive heart failure. Eur Heart J 2000;21:1204-1206.

31 Monahan BP, Ferguson CL, Killeavy ES, Lloyd BK, Troy J, Cantilena LR Jr: Torsades de pointes occurring in association with terfenadine use. JAMA 1990;264:2788-2790.

32 Kothari SS, Sharma S, Wasir HS: Terfenadine overdose induced polymorphous ventricular tachycardia. Indian Pediatr 1995;32:1107-1108.

33 Wang L, Wu T: Torsade De Pointes Caused By Concomitant Use Of Ketoconazole And Terfenadine. Int J Cardiovasc Res 2004;2:5.

-34 Brandenburger M, Wenzel J, Bogdan R, Richardt D, Nguemo F, Reppel M, Hescheler J, Terlau H, Dendorfer A: Organotypic slice culture from human adult ventricular myocardium. Cardiovasc Res 2012;93:50-59. 